\title{
Biomechanical consequences of humeral component malpositioning after anatomical total shoulder arthroplasty
}

\author{
Alexandre Terrier, $\mathrm{PhD}^{\mathrm{a}, *}$, Silvio Ramondetti, MSc ${ }^{\mathrm{a}}$, Francesco Merlini, MSc ${ }^{\mathrm{a}}$, \\ Dominique D. Pioletti ${ }^{a}$, Alain Farron ${ }^{\mathrm{b}}$
}

\author{
${ }^{a}$ Laboratory of Biomechanical Orthopedics, Ecole Polytechnique Fédérale de Lausanne, Lausanne, Switzerland \\ ${ }^{b}$ Service of Orthopaedics and Traumatology, University Hospital Center and University of Lausanne, Lausanne, Switzerland
}

\begin{abstract}
Hypothesis: We hypothesized that the malpositioning of the humeral component can preclude the longterm success of anatomical total shoulder arthroplasty. The goal of this study was to evaluate the mechanical consequences of superior and inferior malpositioning of the humeral head.

Materials and methods: A numerical musculoskeletal model of the shoulder joint allowing natural humeral head translation was used to simulate a loaded abduction movement controlled by muscular activation. An inferior and superior malpositioning of $5 \mathrm{~mm}$ were compared to an optimal positioning. Impingements, articular contact pattern, and cement stress were evaluated.

Results: Inferior malpositioning of the humeral head induced impingement and limited the abduction level, while superior malpositioning increased the subluxation risk. Both inferior and superior malpositioning increased the stress level within the cement mantle.

Discussion: This numerical study highlights the importance of an anatomical reconstruction of the glenohumeral surfaces for the success rate of anatomical total shoulder arthroplasty.

Level of evidence: Basic Science Study.

(C) 2010 Journal of Shoulder and Elbow Surgery Board of Trustees.
\end{abstract}

Keywords: Total shoulder arthroplasty; malpositioning; humeral head; mobility; impingements; biomechanics

Total shoulder arthroplasty with anatomical prostheses is a common and satisfactory treatment for glenohumeral arthritis. A review paper with an average duration of follow-up of 5.3 years reported that overall patient satisfaction was excellent, good, or satisfactory. ${ }^{1}$ The authors of this paper, however, reported that the 2 most common complications are glenoid component loosening (prevalence between 0 and

*Reprint requests: Alexandre Terrier, $\mathrm{PhD}$, Laboratory of Biomechanical Orthopedics, Ecole Polytechnique Fédérale de Lausanne, Station 15, 1015 Lausanne, Switzerland.

E-mail address: alexandre.terrier@epfl.ch (A. Terrier).
$100 \%$ ) and instability (prevalence of $4 \%$ ). In addition, the loosening of the glenoid component has been associated with malpositioning of the prosthesis. ${ }^{25}$ Implant malpositioning, in the sense of a nonanatomical reconstruction of the articular surfaces, can be caused by technical errors in the positioning of anatomical prostheses, but also by using nonanatomical prosthesis designs. ${ }^{12}$

The effect of malpositioning of the humeral head on impingements was evaluated in a cadaveric study. ${ }^{24}$ Offsets in the superior-inferior and anterior-posterior directions were investigated using passive rotation of the humerus at $90^{\circ}$ of arm elevation. In a cadaver study, Nyffeler et al 
measured the effect of superior malpositioning the humeral head on the range of motion and moment arms of the rotator cuff muscles during passive glenohumeral abduction in the scapular plane. ${ }^{11}$ They observed a diminution of the maximum abduction angle and a diminution of the moment arms during the entire range of abduction. In a recent study on 60 cadaveric humeri, ${ }^{13}$ it was shown that impingement was avoided with some third generation prostheses. Positioning was also investigated in a computer model. ${ }^{4}$ Several positioning parameters of the glenoid and humeral components were tested. This study concluded that the height of the humeral head on the resection plane was the most critical parameter of the humeral component. It was reported as the second cause of impingement, after the inclination of the glenoid.

There are thus several efforts to better estimate the consequences of the malpositioning of the humeral component, but these analyses are still limited to impingement problems only. They indeed do not consider the glenohumeral stability or the stress state within the polyethylene or cement mantle. Besides, the current studies used either ball-and-socket numerical models, prohibiting the natural humeral head translation, or use passive cadaver models, which do not account for the compressive and stabilizing effect of the rotator cuff muscles.

Therefore, the goal of this study is to evaluate the biomechanical consequences of a malpositioning of the humeral head component after an anatomical total shoulder arthroplasty. Active abduction in the scapular plane was simulated using a numerical musculoskeletal model of the shoulder accounting for the natural translation of the humeral head in the glenoid fossa. We considered an inferior-superior malpositioning and estimated the impingements, muscle moment arms, muscle forces, joint reaction force, joint contact pattern, and the stress within the polyethylene glenoid component and surrounding cement mantle.

\section{Materials and methods}

A 3-dimensional (3-D) numerical musculoskeletal model of the glenohumeral joint was used for this study (Figure 1). This model is composed of the scapula, humerus, middle deltoid (MD), anterior deltoid (AD), posterior deltoid (PD), supraspinatus (SS), subscapularis (SC), and infraspinatus combined with the teres minor (IS). The geometry of the scapula and humerus were obtained from CT images of a normal cadaveric shoulder. The local mechanical properties of the scapula were obtained from the same CT images, using the relationship between Hounsfield number and elastic modulus. ${ }^{3,9,15}$ Muscles were represented partly by rubber-like structure and cables. The rubber-like part was a 3-D soft deformable rectangular shape embedded with stiff cables, to mimic the mechanical behavior of tendons: very low bending resistance, but high tensile stiffness. The contact between the muscles and the bones was considered to replicate the deformation and wrapping of the muscles around the humeral head. This gave

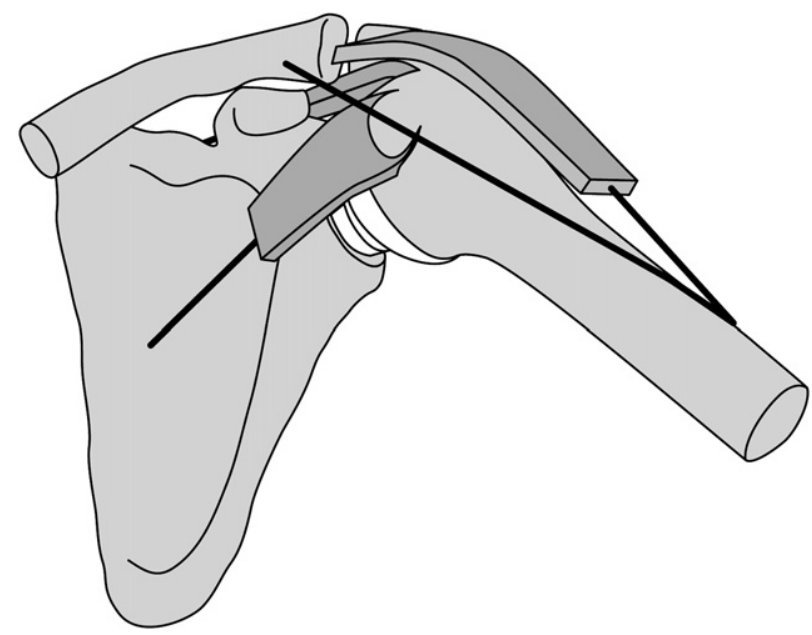

Figure 1 Schematic illustration of the numeric musculoskeletal model used in this study.

the anatomical orientation of the contraction part of the muscle forces, but also the compressive resultant of the muscle force on the humeral head, which provides the stabilization of the joint. The stabilizing effect of the muscles coupled with the glenohumeral contact allowed the natural translation of the humeral head within the glenoid fossa. The arm motion was actively controlled by the muscular activation. A feedback algorithm automatically predicted the muscular forces required balance the arm weight and produce abduction in the scapular plane. This algorithm assumed fixed ratios of the muscular forces, derived from EMG and PCSA measurements, as proposed by Poppen and Walker. ${ }^{14}$ To achieve an elevation of the arm, a shortening of the middle deltoid is imposed. The force induced within the middle deltoid is then considered as the reference force to set the force within the other muscles, according to the predefined muscle ratios. Changing the force within the other muscles obviously alters the reaction force within the middle deltoid (the reference force), the joint reaction force and the overall stability position of the joint. This feedback process was continuously activated while solving the mechanical equilibrium, contact stability, and muscle ratio constraint. Details of this algorithm can be found in 2 previous method papers ${ }^{20,21}$ and 3 application papers. ${ }^{17-19}$

The Aequalis anatomic prosthesis (Tornier Inc, Edina, MN) was inserted into the numerical model according to manufacturer recommendations. The articular surfaces of each component were spherical, with radius of curvature of $30 \mathrm{~mm}$ for the glenoid and $24 \mathrm{~mm}$ for the humeral head. Three configurations were compared: an optimal positioning, an inferior malpositioning of the humeral head, and a superior malpositioning of the humeral head. For the optimal positioning, the humeral head component optimally fitted the intact humeral head. The same method was applied for the glenoid component, with a minimum resection of the glenoid subchondral bone. The inferior malpositioning was simulated by displacing the humeral head by $5 \mathrm{~mm}$ in the inferior direction, along the axis formed by the intersection of the optimal resection plane and the symmetry plane of the prosthesis (in its optimal position). The superior malpositioning was simulated by displacing the humeral head along the same axis, in the superior direction (Figure 2). The positioning of the glenoid and humeral components were assessed visually by a senior orthopaedic 


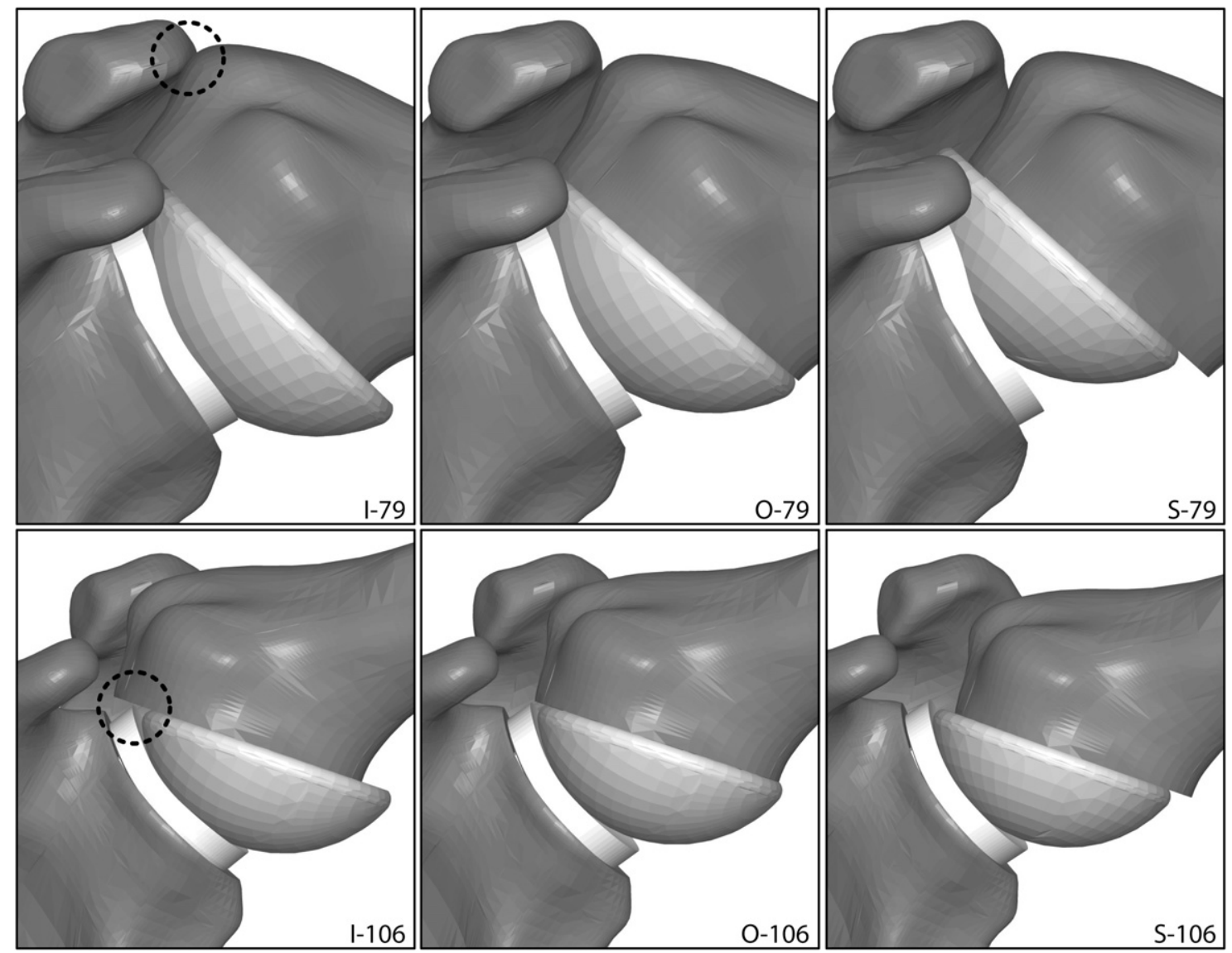

Figure 2 These images come directly from the numerical model and correspond to mechanical equilibrium positions of the humeral head relative to the glenoid at different abduction angles. For clarity, muscles were removed from the display. The first row corresponds to $79^{\circ}$ of abduction, for the inferior (I-79), optimal (O-79), and superior positioning (S-79). With the inferior positioning, there was a slight impingement between the greater tuberosity and the acromion (dotted circle). The second row corresponds to $106^{\circ}$ of abduction. With the inferior positioning, there was a severe impingement between the glenoid component and the superior-lateral border of the humerus (dotted circle), but no impingement with the optimal or superior positioning.

surgeon, using superposition of CT images with the positioned implants. For all configurations, a uniform layer of $0.5 \mathrm{~mm}$ of cement was placed between the host bone and implants. The polyethylene glenoid component and cement layer were considered homogeneous elastic (polyethylene: $\mathrm{E}=500 \mathrm{MPa}, v=0.4$; cement: $\mathrm{E}=2000 \mathrm{MPa}, v=0.3$ ). The virtual reaming, cementing, and positioning of the implants were performed with the standard modelling tools of the computer-aided design software SolidWorks (Dassault Systèmes; SolidWorks, Concord, MA).

A quasi-static movement of arm elevation in the plane of the scapula was simulated from neutral elevation to $150^{\circ}$ of abduction, with full extension of the elbow. The arm weight was $37.5 \mathrm{~N}$ ( $5 \%$ of the body weight of a $75 \mathrm{~kg}$ person) and $10 \mathrm{~N}$ were added in the hand. The motion of the scapula was accounted for to provide the correct alignment of the muscle forces relative to the vertical direction of the arm weight. A scapulo-humeral rhythm of 2:1 was assumed. This means that at $90^{\circ}$ of arm abduction, the scapulothoracic angle was $30^{\circ}$ and the scapulo-humeral angle was $60^{\circ}$.

All numerical analyses were performed with the finite element solver Abaqus 6.7 (www.simulia.com). Linear hexahedral elements were used for the glenoid component and cement mantle, while (modified) quadratic tetrahedral elements were used for the glenoid bone. No friction was considered at the contact between the 2 articular surfaces. The polyethylene-cement and bonecement interfaces were fully bounded.

For the 3 tested configurations, we evaluated the impingement positions, muscle moment arms, muscle forces, joint reaction force, joint contact pattern, and (maximum principal) stress within the cement. The moment arms and stress analysis were only performed every $30^{\circ}$ of abduction, while a continuous evaluation was obtained for the impingement analysis. Impingement positions were evaluated visually by the overlapping of the solid structures.

\section{Results}

When the humeral head was optimally positioned, there was no impingement during the elevation of the arm. When the humeral head was displaced inferiorly, a small impingement (less than $1 \mathrm{~mm}$ penetration) occurred at $79^{\circ}$ of abduction between the greater tuberosity and acromion (Figure 2). After this first slight impingement, there was a more severe impingement at $106^{\circ}$ of abduction between 
the glenoid component and superior-lateral border of the humerus. The superior malpositioning did not induce any impingement problem for the simulated movement. Because elevation was limited to $106^{\circ}$ for 1 of the 3 cases analyzed here, we restricted the comparison of the 3 configurations to this elevation angle.

In overall, the inferior malpositioning of the humeral head increased muscle moment arms, while they were decreased by a superior malpositioning, compared to the reference optimal positioning. This effect was particularly evident at $60^{\circ}$ of elevation (Figure 3). For this position, the 5 -mm inferior (resp. superior) displacement of the humeral head induced approximately 5-mm increase (resp. decrease) of the muscle moment arms. Still, for this position, the change in moment arms induced an overall muscle force decrease of $22 \%$ with the inferior malpositioning and an increase of $77 \%$ with the superior malpositioning. At the joint, the reaction force at $60^{\circ}$ of elevation reached $122 \%$ BW $(915 \mathrm{~N})$ with the optimal case, but was about $16 \%$ lower with the inferior malpositioning and $60 \%$ higher with the superior malpositioning.

With the optimal positioning, the contact pattern was located at the inferior border of the glenoid at the beginning of elevation. It displaced in the superior-posterior quarter of the glenoid surface during the first $30^{\circ}$ of abduction, and then came back to the inferior border during the rest of the elevation, being rather centred at $90^{\circ}$ of abduction (Figure 4). With the inferior malpositioning, the contact pattern displaced to the lower edge of the glenoid component and remained below the lower half of the glenoid surface during the elevation of the arm. With the superior positioning, the contact pattern reached the superiorposterior border of the glenoid component before $30^{\circ}$ of abduction. It remains there until $60^{\circ}$ of abduction. For this position, the humeral head was in a subluxation position. At $90^{\circ}$ of abduction, the contact pattern was again more centered. The average contact pressure on the polyethylene component was maximal at $60^{\circ}$ of abduction. It reached nearly $40 \mathrm{MPa}$ with the superior positioning, while it was below $13 \mathrm{MPa}$ with the optimal and inferior positioning.

Within the cement mantle, the maximal stress value occurred at $60^{\circ}$ of abduction. It remained below $9 \mathrm{MPa}$ with the optimal positioning, but reached $17 \mathrm{MPa}$ and $18 \mathrm{MPa}$ with the inferior and superior malpositioning, respectively. The volumetric distribution of the stress within the cement mantle also confirmed that the stress state was the most critical at $60^{\circ}$ of abduction. For that position, $2.8 \%$ of the cement volume exceeded $5 \mathrm{MPa}$ with the optimal positioning, but it reached $7.4 \%$ and $7.8 \%$ with the inferior and superior malpositioning, respectively (Figure 5).

\section{Discussion}

Although anatomical total shoulder arthroplasty is a common surgical technique to treat various pathologies of the shoulder
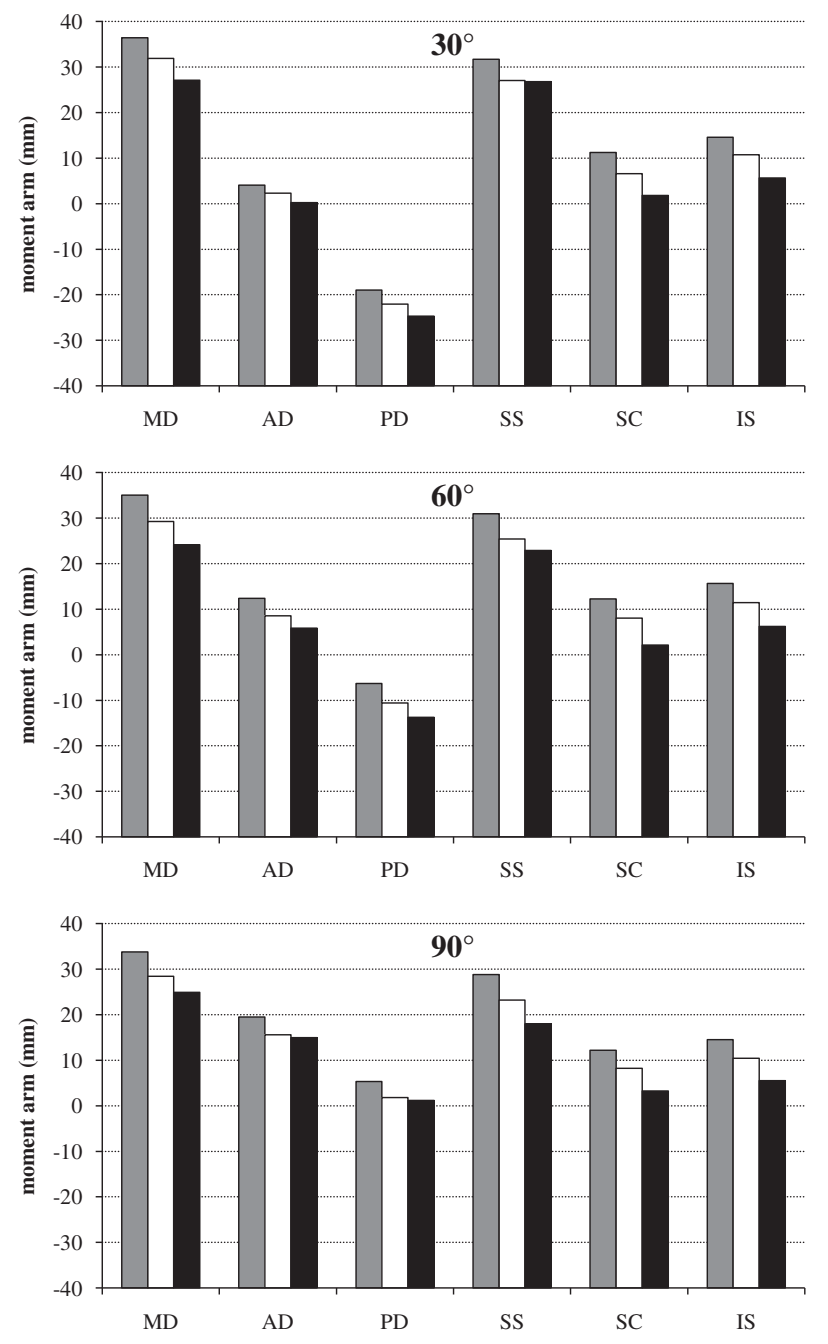

Figure 3 Moment arms of the 6 muscles at 30,60, and $90^{\circ}$ of abduction for the inferior (grey), optimal (white), and superior positioning (black). Overall, muscle moment arms increased with the inferior positioning and decreased with the superior positioning.

such as osteoarthritis, there is a relatively important rate of failure caused by the loosening of the glenoid component, which still requires efforts to limit its occurrence. The correct positioning of the prosthesis components is a key to success of the technique. The anatomical articular surfaces should be reproduced as closely as possible, in order to restore mobility and limit implant loosening. ${ }^{2,12,13}$ The goal of this study was to evaluate the biomechanical consequences of a malpositioning of the humeral component in the inferior-superior direction. The results showed that a relatively small positioning error of $5 \mathrm{~mm}$ can have dramatic effects on the functional outcome (impingement and subluxation) and lifetime of the prosthesis (polyethylene and cement damages). Inferior and superior malpositioning of the humeral head indeed completely altered the normal anatomical biomechanics of the prosthesis, even during a simple elevation movement. 


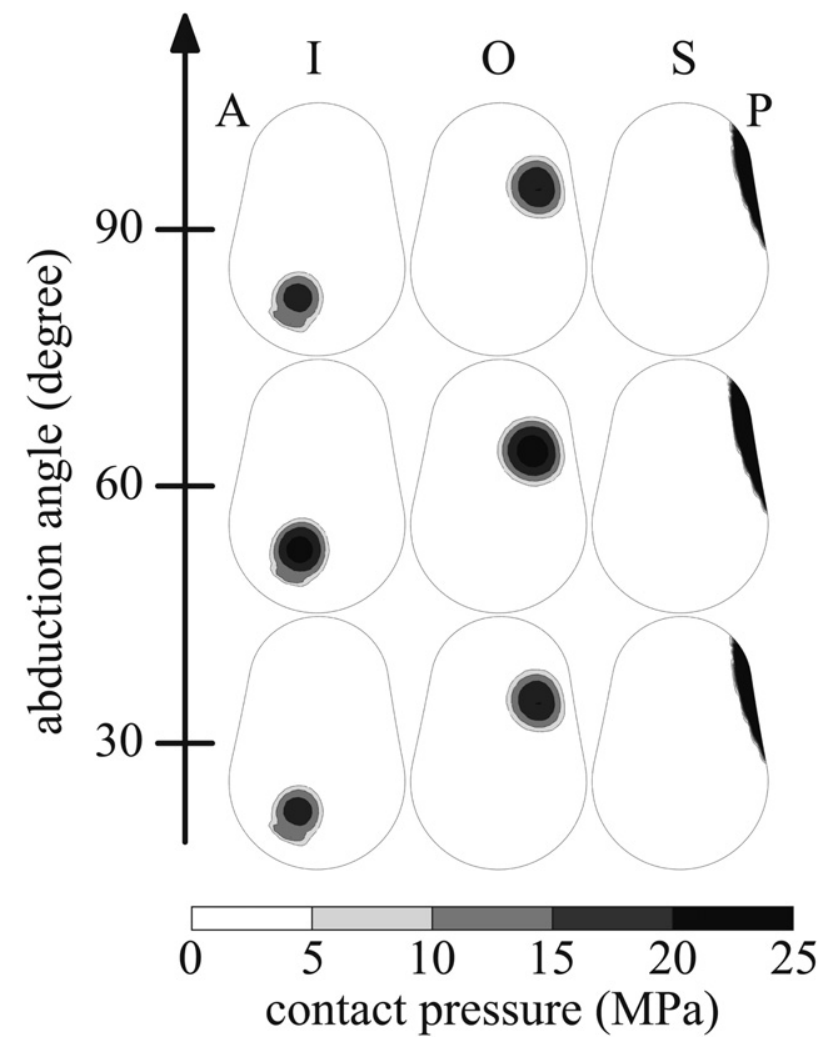

Figure 4 Contact pressure on the glenoid surface during abduction for the inferior ( $I$ : left column), optimal ( $O$ : middle column), and superior positioning ( $S$ : right column) at 30,60 , and $90^{\circ}$ of abduction.

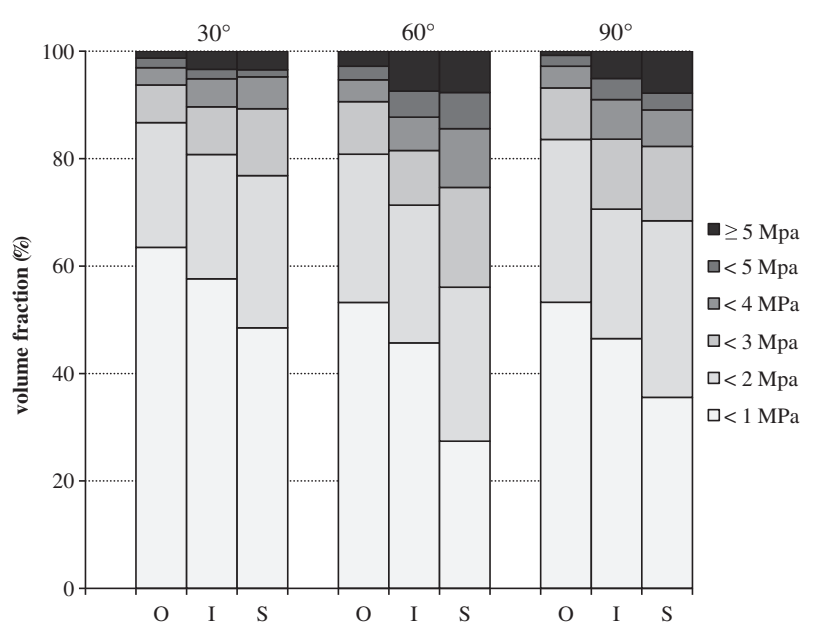

Figure 5 Volumetric distribution of the stress within the cement mantle at 30,60 , and $90^{\circ}$ of abduction, with the optimal $(O)$, inferior $(I)$, and superior $(S)$ positioning.

The risk of impingement may exist after a reconstruction of the shoulder joint. It may reduce the mobility and limit the function of the joint. Our results predict an impingement problem only when the humeral head is displaced inferiorly. This is intuitively expected, as it corresponds to a superior displacement of the humerus relative to its center of rotation. The impingement between the greater tuberosity and the acromion is not surprising. The second impingement between the glenoid component and superiorlateral border of the humerus was also expected. Using a numerical model preventing humeral head translation, Favre et al. observed that an inferior translation of $5 \mathrm{~mm}$ of the humeral head within the resection plane limited the glenohumeral motion to $20^{\circ}{ }^{4}$ For the same malpositioning, our model predicted a similar impingement at $79^{\circ}$ of humerothoracic angle, which corresponds roughly to $50^{\circ}$ of glenohumeral angle. This higher angle before impingement can probably be explained by a lower $(0.2-\mathrm{mm})$ humeral head migration (predicted by the current model but not presented in the results) with the inferior positioning, compared to the optimal positioning $(1 \mathrm{~mm})$ during elevation up to the impingement at $79^{\circ}$. The study of Favre et al also concluded that the superior malpositioning did not induce impingement, at least with a physiological range of motion. ${ }^{4}$ Using a cadaveric model, inferior-superior malpositioning of the humeral head was also investigated. ${ }^{24} \mathrm{~A}$ passive rotation of the humerus was applied while the humerus was at $90^{\circ}$ of elevation, corresponding to $60^{\circ}$ of glenohumeral elevation. For superior and inferior malpositioning of $8 \mathrm{~mm}$, the range of internal-external rotation was reduced by about $10^{\circ}$, but was mildly affected by 4-mm offsets. The authors of this study observed that small inferior offset can lead to significant increase in subacromial contact, which was not observed for superior offset. In another cadaver experiment, Nyffeler et al observed no mechanical impingement with a superior malpositioning of $10 \mathrm{~mm}$, but reported a reduction of about $10^{\circ}$ in the range of gleno-humeral abduction caused by the tensioning of the inferior capsuloligamentous structures. ${ }^{11}$ The same study reported also changes in moment arms of the muscles that are consistent with our predictions. Another cadaveric study reported that the subacromial space was minimal at $90^{\circ}$ of abduction. ${ }^{5}$ Although we did not actually measure subacromial space, we observed (Figure 2, O-79 and O-106, corresponding, respectively, to 79 and 106 degrees of elevation) that the minimum subacromial space was indeed in between, which is consistent with the above paper.

As expected, the muscle forces changed in the opposite way to moment arms, to maintain the same level of moment of force to balance the arm weight. In overall, the muscular forces predicted here are in the same order of other cadaveric or numerical models, although there are important differences among them. The joint reaction force, being the vectorial summation of all forces applied to the humerus (muscles, arm weight, external force in the hand), also decreased with the inferior displacement and increased with the superior displacement of the humeral head. Overall, the reaction force predicted here was in good agreement with recent in vivo measurements. ${ }^{23}$ Although a strict comparison is difficult, lifting a weight of $1.5 \mathrm{~kg}$ with a straight arm produced a reaction force between 90 
and $125 \%$ bodyweight, which is rather close to the $122 \%$ of the bodyweight predicted here for an external force of $10 \mathrm{~N}$ in the hand.

The contact pattern of the optimal case is consistent with the literature. This up and down movement of the contact pattern has been reported in other numerical model, cadaveric studies, and in vivo measurements. ${ }^{10,16,22}$ The inferior (resp. superior) displacement of the contact pattern when the humeral head is inferiorly (resp. superiorly) displaced has not been reported yet; but, this behavior is rational and can be explained as follows. The inferiorsuperior malpositioning of the humeral head changes the direction of the muscle forces relatively to the glenoid, which also change in the opposite direction the reaction force on the glenoid surface. Inferior displacement of the humeral head induces a resultant muscle force more inferiorly directed and a reaction force more superiorly directed, which, being more or less perpendicular to the articular surface, will be applied more inferiorly; and produce the predicted more inferior contact pattern. The opposite reasoning is applicable for the superior malpositioning, producing a more superior contact pattern. In the case of the superior malpositioning, the contact pattern was also displaced towards the posterior border of the glenoid. This is because of the orientation of the glenoid component, which follows the natural orientation of the glenoid to have the best bone support. We thus observed that the superior malpositioning of the humeral head is more critical regarding the joint stability.

Cement stress predictions showed that both inferior and superior malpositioning of the humeral head induced an increase of the maximum, but also the volume of stress above some critical value. The critical value to be considered could be the endurance (or fatigue) limit of the cement, which has been reported to be about $7 \mathrm{MPa}^{8}$ Our results (Figure 5) predict 2-fold volume of cement exceeding this limit when the humeral head is inferiorly or superiorly malpositioned by $5 \mathrm{~mm}$.

The strength of the present study is to account for the natural translation of the humeral head during a loaded movement of the shoulder actively controlled and stabilized by the muscles. This translation of the humeral head during arm elevation has already been observed in vitro and in vivo, and our predictions are consistent with thses reported data. ${ }^{6,7}$ Although the present analysis is limited to arm elevation in the scapular plane, this movement is biomechanically relevant for most activities of daily living, as it is assumed by most cadaveric studies. Fixed muscular ratios were used to simulate this movement for the 3 cases; however, the muscular forces were different in each case. The change of the articular configuration also changed the equilibrium positions, muscle moment arms, and thus the muscular forces. The capsule was not accounted for in this model, but we assumed that its effect was negligible for the simulated range of abduction. Only inferior-superior malpositioning was considered, but this is the most common positioning error during total shoulder arthroplasty. Finally, no damaging behavior of the materials was accounted for, such as plastic (permanent) deformation of the polyethylene or fractures of the cement, which could better predict the failure risk of the malpositioning. The finite element method can be very efficient to analysis the stress state within prosthesis components; but, the method and its predictions need to be carefully verified, especially when the method is applied to complex biological system. In this work, the main limitation concerns the cement stress, which is obtained for a perfect homogenous layer between the glenoid component and a single healthy glenoid bone. Results might be different with more complex cement distribution and more pathological scapulae. This comparative study, however, clearly reports the relative effect of a humeral component malpositioning.

\section{Conclusion}

To conclude, this biomechanical analysis of the humeral head malpositioning in anatomical total shoulder arthroplasty highlighted and explained its possible consequences on the final outcome of the joint replacement, such as reduced mobility, subluxation, polyethylene wear, cement fracture, and component loosening. Functional result and survivorship of such prostheses are indeed very sensitive to a correct positioning of the components, which should reproduce the anatomical articular surfaces. This work thus confirms the importance of an accurate anatomical reconstruction of the humeral side of total shoulder arthroplasty to restore mobility and to reduce the risk of glenoid loosening.

\section{Disclaimer}

The authors, their immediate families, and any research foundations with which they are affiliated did not receive any financial payments or other benefits from any commercial entity related to the subject of this article.

This study was funded partly by Tornier (Tornier, Inc., Edina, MN) and partly by the Center of Translational Biomechanics EPFL-CHUV-DAL, Lausanne, Switzerland. The authors especially thank Yves-Alain Ratron (Research Director, Tornier) for his help.

\section{References}

1. Bohsali KI, Wirth MA, Rockwood CA Jr. Complications of total shoulder arthroplasty. J Bone Joint Surg Am 2006;88:2279-92. 88/10/ 2279 [pii], doi:10.2106/JBJS.F.00125 
2. Boileau P, Walch G. The three-dimensional geometry of the proximal humerus. Implications for surgical technique and prosthetic design. J Bone Joint Surg Br 1997;79:857-65. doi:10.1302/0301-620X.79B5.7579

3. Carter DR, Hayes WC. The compressive behavior of bone as a twophase porous structure. J Bone Joint Surg Am 1977;59:954-62.

4. Favre P, Moor B, Snedeker JG, Gerber C. Influence of component positioning on impingement in conventional total shoulder arthroplasty. Clin Biomech (Bristol, Avon) 2008;23:175-83. S0268-0033(07) 00210-0 [pii], doi:10.1016/j.clinbiomech.2007.09.009

5. Flatow EL, Soslowsky LJ, Ticker JB, Pawluk RJ, Hepler M, Ark J, et al. Excursion of the rotator cuff under the acromion. Patterns of subacromial contact. Am J Sports Med 1994;22:779-88.

6. Graichen H, Stammberger T, Bonel H, Haubner M, Englmeier KH, Reiser M, et al. Magnetic resonance-based motion analysis of the shoulder during elevation. Clin Orthop 2000:154-63.

7. Kelkar R, Wang VM, Flatow EL, Newton PM, Ateshian GA, Bigliani LU, et al. Glenohumeral mechanics: a study of articular geometry, contact, and kinematics. J Shoulder Elbow Surg 2001;10:73-84.

8. Lewis G. Properties of acrylic bone cement: state of the art review. J Biomed Mater Res 1997;38:155-82. doi:10.1002/jbm.b.31220

9. Mansat P, Barea C, Hobatho MC, Darmana R, Mansat M. Anatomic variation of the mechanical properties of the glenoid. J Shoulder Elbow Surg 1998;7:109-15.

10. Massimini DF, Li G, Warner JJ. Glenohumeral articular contact kinematics of patients after total shoulder arthroplasty. In: 54th Annual Meeting of the Orthopaedic Research Society; 2008, San Francisco; 2008.

11. Nyffeler RW, Sheikh R, Jacob HA, Gerber C. Influence of humeral prosthesis height on biomechanics of glenohumeral abduction. An in vitro study. J Bone Joint Surg Am 2004;86A:575-80.

12. Pearl ML, Kurutz S. Geometric analysis of commonly used prosthetic systems for proximal humeral replacement. J Bone Joint Surg Am 1999;81:660-71.

13. Pearl ML, Kurutz S, Postachini R. Geometric variables in anatomic replacement of the proximal humerus: how much prosthetic geometry is necessary? J Shoulder Elbow Surg 2009;18:366-70. S1058-2746(09) 00076-7 [pii], doi:10.1016/j.jse.2009.01.011

14. Poppen NK, Walker PS. Forces at the glenohumeral joint in abduction. Clin Orthop 1978:165-70.

15. Rho JY, Hobatho MC, Ashman RB. Relations of mechanical properties to density and CT numbers in human bone. Med Eng Phys 1995; 17:347-55. doi:10.1016/1350-4533(95)97314-F
16. Soslowsky LJ, Flatow EL, Bigliani LU, Pawluk RJ, Ateshian GA, Mow VC. Quantitation of in situ contact areas at the glenohumeral joint: a biomechanical study. J Orthop Res 1992;10:524-34. doi:10. 1002/jor. 1100100407

17. Terrier A, Merlini F, Pioletti DP, Farron A. Comparison of polyethylene wear in anatomical and reversed shoulder prostheses. J Bone Joint Surg Br 2009;91:977-82. 91-B/7/977 [pii], doi:10.1302/0301620X.91B7.21999

18. Terrier A, Merlini F, Pioletti DP, Farron A. Total shoulder arthroplasty: Downward inclination of the glenoid component to balance supraspinatus deficiency. J Shoulder Elbow Surg 2009;18:360-5. doi:10. 1016/j.jse.2008.11.008

19. Terrier A, Reist A, Merlini F, Farron A. Simulated joint and muscle forces in reversed and anatomic shoulder prostheses. J Bone Joint Surg Br 2008;90:751-6. 90-B/6/751 [pii], doi:10.1302/0301-620X.90B6. 19708

20. Terrier A, Reist A, Vogel A, Farron A. Effect of supraspinatus deficiency on humerus translation and glenohumeral contact force during abduction. Clin Biomech 2007;22:645-51. doi:10.1016/j.clinbiomech. 2007.01.015

21. Terrier A, Vogel A, Capezzali M, Farron A. An algorithm to allow humerus translation in the indeterminate problem of shoulder abduction. Med Eng Phys 2008;30:710-6. S1350-4533(07)00148-8 [pii], doi:10.1016/j.medengphy.2007.07.011.

22. van der Helm FC. Analysis of the kinematic and dynamic behavior of the shoulder mechanism. J Biomech 1994;27:527-50. doi:10.1016/ 0021-9290(94)90064-7

23. Westerhoff P, Graichen F, Bender A, Halder A, Beier A, Rohlmann A, et al. In vivo measurement of shoulder joint loads during activities of daily living. J Biomech 2009;42:1840-9. S0021-9290(09)00316-9 [pii], doi:10.1016/j.jbiomech.2009.05.035

24. Williams GR, Wong KL, Pepe MD, Tan V, Silverberg D, Ramsey ML, et al. The effect of articular malposition after total shoulder arthroplasty on glenohumeral translations, range of motion, and subacromial impingement. J Shoulder Elbow Surg 2001;10:399-409. doi:10.1067/ mse.2001.116871

25. Wirth MA, Ondrla J, Southworth C, Kaar K, Anderson BC, Rockwood CA III. Replicating proximal humeral articular geometry with a third-generation implant: a radiographic study in cadaveric shoulders. J Shoulder Elbow Surg 2007;16:S111-6. doi:10.1016/j.jse. 2006.09.008 